\title{
Structure of baryons in a relativistic quark model
}

\author{
Bernard Metsch $^{\mathrm{a}}$ \\ ${ }^{\text {a }}$ Helmholtz-Institut für Strahlen- und Kernphysik (Theorie), \\ Universität Bonn, Nußallee 14-16, D-53115 Bonn, Germany
}

Baryonic excitation spectra, electroweak and strong decay properties are discussed within a relativistically covariant constituent quark model based on the instantaneous approximation to the three-body Bethe-Salpeter equation.

\section{Introduction}

Baryonic resonance spectra exhibit some striking features: Linear Regge-trajectories, which hint at a linear confinement potential; moderately large hyperfine splittings (e.g. the $N-\Delta$-splitting) hinting at a strong spin-spin interaction; parity doublets, such as e.g. $N_{\frac{5}{2}}^{*}+(1680)-N_{\frac{5}{2}}^{*}(1675)$, which all are a challenge to explain theoretically. The most successful approaches to account for these have been constituent quark models (in nonrelativistic or 'relativized' versions), see e.g. the excellent review by Capstick and Roberts [ 1] and references therein, which use one-gluon-exchange or Goldstone-boson-exchange as quark interaction in addition to a linear confinement potential. Although the results from such calculations are in general satisfactory, they do not reproduce the details of the $N$-Regge-trajectory nor explain the parity doublets found. Moreover, the role of the spinorbit parts of the residual interactions remains obscure. On top of this the conventional constituent quark models have no real field theoretical basis and lack relativistic covariance. As an extension of an earlier relativistic quark model description of mesons [2], we therefore developed a relativistic quark-model for baryons on the basis of the three-particle Bethe-Salpeter equation.

The details of our approach are extensively described in [3], here we shall merely quote the basic assumptions and features. Starting point is the Bethe-Salpeter-Equation for bound states of three fermions, which is a homogeneous integral equation involving full quark propagators and irreducible interaction kernels in terms of the 8 relative momentum variables of the quarks. In order to solve this equation we assumed, inspired by the apparent success of the non-relativistic constituent quark model, that the self-energy in the quark propagators can be suitably approximated by introducing an effective, constituent quark mass in the free Feynman-propagator and, furthermore, that the interaction kernels do not depend on the relative energy variables of the quarks in the rest-frame of the baryon. Although this also implies a technical simplification (Salpeter equation), the main reason is that we want to implement confinement by an instantaneous linearly rising three-body potential. These assumptions, after introducing an effective instantaneous kernel which approximates retardation effects in two-body interactions, allow for a formulation of the 
resulting Salpeter equation as an eigenvalue equation, which is solved by expanding the amplitudes in a suitable large, but finite, basis.

\section{The model}

Confinement is implemented by a string-like three body potential, which rises linearly with inter-quark distances and comprises a spin structure which was chosen such, that spin-orbit splittings are suppressed, see [4] for details. In order to account for the hyperfine structure we adopted an effective two-body interaction based on instanton effects, which has the decisive property to solve the $U_{A}(1)$-problem in the pseudoscalar meson spectrum [2]. For two quarks it is a short-range two-body interaction acting on quark pairs with vanishing spin which are antisymmetric in flavour. Consequently this force does not act on the flavour symmetric $\Delta$-resonances. The Regge-trajectory in this sector is then used to determine the (non-strange) constituent quark mass and the constant and string tension parameters of the confinement potential. The three parameters of the instanton force are determined from the ground-state octet-decuplet splittings. The remaining spectrum is then a genuine prediction.

\section{Mass spectra}

The resulting mass spectra, see e.g. Fig. 1 for non-strange and strange baryons can be found in [4] and [5], respectively. In general a very satisfactory description of the masses of states up to $2.5 \mathrm{GeV}$ is found. The most prominent features are: Once the strengths of the instanton induced interaction are fixed from the ground state splittings the other prominent hyperfine splittings in the spectrum can be explained quite naturally: In contrast to earlier non-relativistic quark model calculations with the same interaction, in the present relativistic setup the instanton-induced interaction is strong enough to account almost quantitatively for the low position of the Roper-resonances and its analogues in the strange sectors. In addition, the $N$ - and $\Lambda$ - Regge trajectory can be very nicely reproduced, indicating that this force leads to a constant shift in $M^{2}$ for these states, in accordance with experimental data. Moreover this force also accounts for the occurrence of the parity doublets mentioned above: selectively those states from a particular shell (in the harmonic oscillator classification), which show scalar diquark configurations are lowered enough to become degenerate with some states of the lower oscillator shell with opposite parity [4].

\section{Electromagnetic observables}

On the basis of the Salpeter amplitudes, the baryonic vertex function(amputated BetheSalpeter amplitude) can be reconstructed for any on-shell baryon momentum und thus form factors and various couplings can be calculated covariantly within the Mandelstam formalism, in impulse approximation without any additional parameters. The details and results of the calculation of static moments, electroweak form factors and photon couplings of non-strange baryons have been published recently [ 8]. Here we will briefly discuss a novel approach to calculate magnetic moments directly from the Salpeter amplitudes and some new results for semi-leptonic and strong decays. 


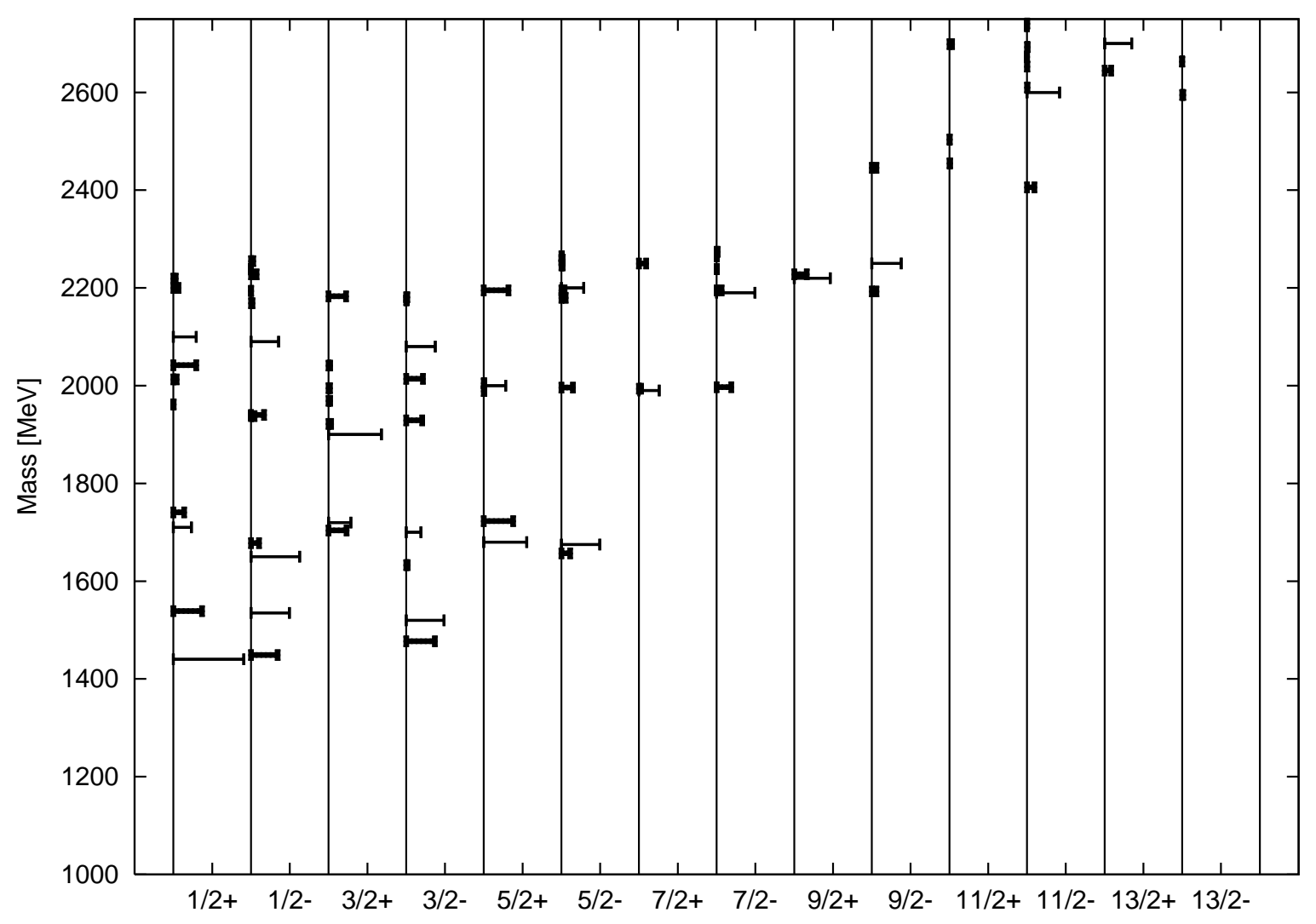

Figure 1. Decay amplitudes (proprtional to the square root of the partial decay width) of strong $N^{*} \rightarrow N \pi$ decays. In each column (i.e. for each spin and parity $J \pi$ ) the experimental value (thin horizontal bars at the experimaental resonance position) is compared to the calculated value (thick horizontal bars at the calculated resonance position).

\subsection{Magnetic moments}

The interaction energy of a baryon in an external electromagnetic field is given by the matrix element of $\hat{j}_{\mu} A^{\mu}$ where $\hat{j}$ is the electromagnetic current. The magnetic moment is then the coefficient of the term linear to a static magnetic field and can be expressed as

$\mu=-\frac{1}{4 M} \varepsilon_{3 j k} i\left[\frac{\partial}{\partial \bar{P}^{j}}\left\langle\bar{M}, S, S_{3}=S\left|j^{k}\left(x^{0}, \overrightarrow{0}\right)\right| \bar{P}, S, S_{3}=S\right\rangle\right]_{\bar{P}=0}$,

where $|\bar{M}, S\rangle$ and $|\bar{P}, S\rangle$ involve the vertex functions of a baryon with mass $M$ and spin $S$ in the rest frame and of a baryon with momentum $\bar{P}$, respectively. Evaluating this expression we then find, that in impulse approximation the magnetic moment can be expressed in momentum space as the expectation value of a local operator $\hat{\mu}$ with respect to the Salpeter amplitudes $\Phi_{M}^{\Lambda}$, normalized to $\left\langle\Phi_{M}^{\Lambda} \mid \Phi_{M}^{\Lambda}\right\rangle=2 M$ :

$\mu=\frac{\left\langle\Phi_{M}^{\Lambda}|\hat{\mu}| \Phi_{M}^{\Lambda}\right\rangle}{2 M}$, where $\hat{\mu}^{3}=\frac{\omega_{1}+\omega_{2}+\omega_{3}}{M}\left(\sum_{\alpha=1}^{3} \frac{\hat{e}_{\alpha}}{2 \omega_{\alpha}}\left(\hat{\ell}_{\alpha}^{3}+\hat{\Sigma}_{\alpha}^{3}\right)\right)-\hat{\mu}_{C}^{3}$, 
where $\omega_{\alpha}=\sqrt{m_{\alpha}^{2}+p_{\alpha}^{2}}$ represents the relativistic energy of quark $\alpha$ with mass $m_{\alpha}$ and charge $\hat{e}_{\alpha}, \hat{\ell}$ and $\hat{\Sigma}$ represent the single particle angular momentum- and (twice the) spin-operator, respectively, and where $\hat{\mu}_{C}^{i}=\varepsilon_{i j k} \frac{1}{M} \sum_{\alpha_{0}}^{3} \frac{\hat{e}_{\alpha}}{2 \omega_{\alpha}} p_{\alpha}^{k} \sum_{\beta=1}^{3} \omega_{\beta} \frac{\partial}{\partial p_{\beta}^{j}}$. corrects for the relativistic center of charge motion. We think that this is a remarkable expression, to our knowledge not to be found in the literature. The non-relativistic limit of this expression is of course obvious. The results for octet baryons are given in Table 1 .

Table 1

Magnetic moments of octet baryons in units of $\mu_{N}$.

\begin{tabular}{lllllr}
\hline B & \multicolumn{1}{c}{ Exp. } & Calc. & B & Exp. & Calc. \\
\hline$p$ & 2.792 & 2.77 & $\Lambda$ & $-0.613 \pm 0.004$ & -0.61 \\
$n$ & -1.913 & -1.71 & $\Sigma^{+}$ & $2.458 \pm 0.010$ & 2.51 \\
$\Xi^{0}$ & $-1.250 \pm 0.014$ & -1.33 & $\Sigma^{0}$ & $(0.649)$ & 0.75 \\
$\Xi^{-}$ & $-0.65 \pm 0.08$ & -0.56 & $\Sigma^{-}$ & $-1.160 \pm 0.125$ & -1.02 \\
\hline
\end{tabular}

The experimental values, except for the value for $\mu_{\Sigma_{0}}$ which is taken via isospin invariance from $\mu_{\Sigma^{+}}$and $\mu_{\Sigma^{-}}$, are given in ref. [ [7].

\subsection{Semi-leptonic decays}

Some new, representative results for semi-leptonic decays, calculated from the weak baryonic currents in the Mandelstam formalism, are listed in Table 2.

\section{Strong decays and the problem of "missing resonances"}

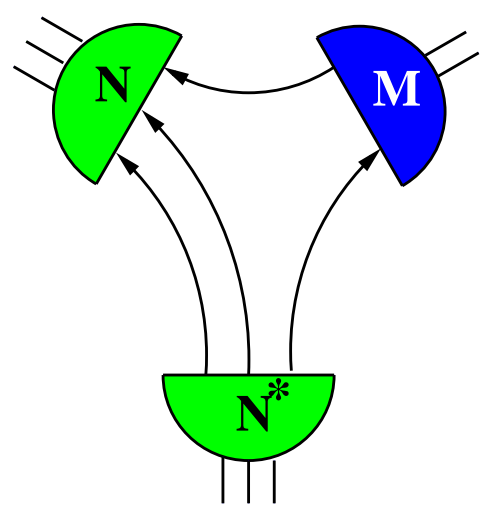

Figure 2. Lowest order quark-loop contribution to the strong $N^{*} \rightarrow N+M$ decay of excited baryons.
In the framework of the Mandelstam formalism the amplitude for the strong mesonic decay of excited baryons can be obtained in lowest order by evaluating the simple quark loop diagram of Fig. 2, which involves the vertex functions (amputated BetheSalpeter-amplitudes) of the participating meson, obtained from a previous calculation on mesons [2], and baryons. Although in general the calculated partial widths are too small to account for the experimental values quantitatively, appreciable decay widths are found only for the well established resonances, the predicted values for higher lying resonances being in general smaller by at least an order of magnitude, see also Fig. 1, thus explaining why these have not been observed so far in elastic pion-nucleon scattering. This observation is in accordance with the findings cited in [1]. 
Table 2

Decay rates and axial vector couplings of semi-leptonic decays of baryons.

\begin{tabular}{|c|c|c|c|c|c|}
\hline & & \multicolumn{2}{|c|}{$\Gamma\left[10^{6} \mathrm{~s}^{-1}\right]$} & \multicolumn{2}{|c|}{$g_{A} / g_{V}$} \\
\hline & Decay & Exp. & Calc. & Exp. & Calc. \\
\hline$n$ & $\rightarrow p e^{-} \bar{\nu}_{e}$ & & & $1.2670 \pm 0.0035$ & 1.21 \\
\hline & $\rightarrow p e^{-} \bar{\nu}_{e}$ & $3.16 \pm 0.06$ & 3.10 & $-0.718 \pm 0.015$ & -0.82 \\
\hline$\Sigma^{+}$ & $\rightarrow \Lambda e^{+} \nu_{e}$ & $0.25 \pm 0.06$ & 0.20 & & \\
\hline$\Sigma^{-}$ & $\rightarrow \Lambda e^{-} \bar{\nu}_{e}$ & $0.38 \pm 0.02$ & 0.34 & & \\
\hline$\Sigma^{-}$ & $\rightarrow n e^{-} \bar{\nu}_{e}$ & $6.9 \pm 0.2$ & 4.91 & $0.340 \pm 0.017$ & 0.25 \\
\hline$\Xi^{0}$ & $\rightarrow \Sigma^{+} e^{-} \bar{\nu}_{e}$ & $0.93 \pm 0.14$ & 0.91 & $1.32_{-0.17}^{+0.21} \pm 0.05$ & 1.38 \\
\hline$\Xi^{-}$ & $\rightarrow \Sigma^{0} e^{-} \bar{\nu}_{e}$ & $0.5 \pm 0.1$ & 0.51 & & \\
\hline$\Xi^{-}$ & $\rightarrow \Lambda e^{-} \bar{\nu}_{e}$ & $3.3 \pm 0.2$ & 2.30 & $-0.25 \pm 0.05$ & -0.27 \\
\hline$\Omega^{-}$ & $\rightarrow \Xi^{0} e^{-} \bar{\nu}_{e}$ & $68 \pm 34$ & 46 & & \\
\hline$\Lambda$ & $\rightarrow p \mu^{-} \bar{\nu}_{\mu}$ & $0.60 \pm 0.13$ & 0.47 & & \\
\hline$\Sigma^{-}$ & $\rightarrow n \mu^{-} \bar{\nu}_{\mu}$ & $3.04 \pm 0.27$ & 1.60 & & \\
\hline$\Xi^{-}$ & $\rightarrow \Lambda \mu^{-} \bar{\nu}_{\mu}$ & $2.1 \pm 1.3$ & 1.04 & & \\
\hline
\end{tabular}

The experimental values are given in ref. [ 7 ].

\section{Conclusion}

In conclusion we think that we have demonstrated, that a fully relativistic treatment of the quark dynamics and their electromagnetic couplings on the basis of the instantaneous Bethe-Salpeter equation with quark forces from a confinement potential and an instantoninduced interaction indeed leads to an encouraging description of the major features of the baryonic excitation spectrum upto $3 \mathrm{GeV}$, including numerous electromagnetic and strong decay observables, This description still can and, in view of the approximate treatment of retardation effects from the two-body interaction, must be improved.

Major contributions by Christian Haupt, Dirk Merten, Sascha Migura and Herbert Petry are gratefully acknowledged. We thank the DFG for financial support.

\section{REFERENCES}

1. S. Capstick, W. Roberts, Prog. Part. Nucl. Phys., 45, (2000) 241.

2. M. Koll, R. Ricken, D. Merten, B. Metsch, H. Petry, Eur. Phys. J. A9, (2000) 73.

3. U. Löring, K. Kretzschmar, B. Metsch, H. Petry, Eur. Phys. J. A10, (2001) 309.

4. U. Löring, B. Metsch, H. Petry, Eur. Phys. J. A10, (2001) 395.

5. U. Löring, B. Metsch, H. Petry, Eur. Phys. J. A10, (2001) 447.

6. S. Capstick, W. Roberts, Phys. Rev D49 (1994) 4570.

7. Particle Data Group, D.E. Groom et al., Eur. Phys. J. C15, (2000) 1.

8. D. Merten, U. Löring, K. Kretzschmar, B. Metsch, H. Petry, Eur. Phys. J. A14, (2002) 477 